\title{
Effects of apoptosis-related proteins caspase-3, Bax and Bcl-2 on cerebral ischemia rats
}

\author{
GUANGYI LIU ${ }^{1}$, TAO WANG ${ }^{2}$, TINGING WANG ${ }^{1}$, JINMING SONG ${ }^{1}$ and ZHEN ZHOU ${ }^{1}$ \\ ${ }^{1}$ Institute of Cerebrovascular Diseases, Affiliated Hospital of Qingdao University Medical College, Qingdao, \\ Shandong 266003; ${ }^{2}$ Department of Neurology, The Central Hospital of Taian, Taian, Shandong 271000, P.R. China
}

Received May 20,2013; Accepted July 03, 2013

DOI: $10.3892 /$ br.2013.153

\begin{abstract}
Neuron apoptosis is known to mediate a change of ethology following cerebral ischemia/reperfusion injury in rats. Additionally, Bcl-2, Bax and caspase-3 proteins may exert a significant effect on neuron injury. The aim of this study was to investigate the role, mechanism of action and clinical significance of these proteins in neuron apoptosis and functional impairment following cerebral ischemia/reperfusion injury in rats. Sixty male healthy adult Wistar rats were randomly assigned into control $(n=6)$, sham operation $(n=6)$ and experimental $(n=48)$ groups. The model of rat cerebral ischemia/reperfusion injury was set up according to the method of Zea-Longa. Eight subsets of 6 rats/subset were designed according to time points (at 3, 6, 12,24 and $48 \mathrm{~h}$ and at 3,7 and 14 days). Nerve functional injury was evaluated and graded using nerve function score, balance, coordination function detection and measurement of forelimb placing. The neurons expressing caspase-3, Bax and Bcl-2 in the cortical area, CA3, CA1, stratum lucidum (Slu) and molecular layer of the dentate gyrus (MoDG) of the hippocampus were detected using immunohistochemistry or the TUNEL method. The expression of caspase-3, Bax and Bcl-2 genes was detected by the reverse transcriptase polymerase chain reaction (RT-PCR). The results indicated that, compared to the sham operation group, the score of nerve function and balance beam walking were distinctly higher $(\mathrm{P}<0.01)$ and the percentage of rat foreleg touching the angle or margin of the table was significantly lower in the experimental rat group $(\mathrm{P}<0.01)$ at $3 \mathrm{~h}$ following reperfusion. The expression of TUNEL-positive neurons was high in the cortical area and the CA3 region of the hippocampus $(\mathrm{P}<0.01)$, caspase-3 was at peak value in the cortical area and the CA1 region of the hippocampus $(\mathrm{P}<0.01)$, Bax was increased in the cortical area and the Slu of the hippocampus $(\mathrm{P}<0.01)$ and $\mathrm{Bcl}-2$ was low in the cortical area and the MoDG of the hippocampus $(\mathrm{P}<0.01)$ in the experimental group
\end{abstract}

Correspondence to: Dr Guangyi Liu, Institute of Cerebrovascular Diseases, Affiliated Hospital of Qingdao University Medical College, 16 Jiangsu Road, Qingdao, Shandong 266003, P.R. China E-mail: 15898877166@163.com

Key words: cerebral ischemia/reperfusion injury, caspase-3, Bax, Bcl-2, neuron apoptosis, neurological impairment at $48 \mathrm{~h}$ following reperfusion. In conclusion, cerebral ischemia/ reperfusion injury may cause neurological impairment and lead to a change of ethology, and neuron apoptosis may be associated with the activation of caspase- 3 and Bax and the downregulation of Bcl-2.

\section{Introduction}

Nerve cell apoptosis may occur following cerebral ischemia or reperfusion injury as a result of the expression of apoptosis-related proteins and a complicated process leading to physiological and pathological alterations. The brain tissue in the ischemic area undergoes irreversible damage within a short period of time and develops foci of infarction. Ischemic semi-diazonal nerve cells principally undergo delayed apoptotic neuronal death (1), which is a key factor leading to motor dysfunction and neurological impairment. Our previous studies $(2,3)$ indicated that the loci of brain tissue damage and pathological characteristics in a rat ischemia model coincided with human cerebral infarction loci and clinical symptoms with regard to injury as the same target tissue is involved in both rats and humans. Loci vulnerable to injury were shown to be the sensitive functional areas of the cortex and hippocampus. Those studies also demonstrated that the expressed Bax activated caspase-3, indirectly inhibited Bcl-2 expression, formed $\mathrm{Bax} / \mathrm{Bcl}-2$ heterodimers and initiated cell apoptosis following cerebral ischemia/reperfusion injury. Those results also confirmed the significance of the cerebral ischemia/reperfusion injury model in clinical diagnosis. The present study investigated the expression of caspase-3, Bcl-2 and Bax and neuron apoptosis, as well as the ethological alterations following cerebral ischemia/reperfusion injury in a rat model.

\section{Materials and methods}

Materials. Sixty healthy, adult, specific pathogen-free (SPF) Wistar rats, with a body weight of 200-240 g, were supplied by the Experimental Animal Center of Shandong University, Jinan, China; the in situ end-labeling TUNEL kit was purchased from Roche Diagnostics GmbH, Mannheim, Germany; the rabbit anti-rat caspase-3 polyclonal antibody was purchased from Santa Cruz Biotechnology, Inc. (Santa Cruz, CA, USA) and the rabbit anti-rat Bax or Bcl-2 monoclonal antibodies were obtained from Sigma-Aldrich (St. Louis, 
MO, USA); the TRIzol kit, streptavidin-biotin-peroxidase complex (SABC) immunohistochemistry kit and 3,3'-diaminobenzidine (DAB) kit were purchased from Wuhan Boshide Biological Engineering Co. Ltd. (Wuhan, China); a BX20 microscope camera was provided by Olympus (Tokyo, Japan); a paraffin microtome was obtained from Microm International GmbH (Walldorf, Germany); the GeneAmp PCR system 2400 was purchased from Perkin Elmer (Waltham, MA, USA); the Bio-Gel Analysis system was purchased from Bio-Rad (Hercules, CA, USA); and the HPIAS-1000 Image Analysis system was purchased from Wuhan Tongji Qianping Image Engineering (Wuhan, China). All animal experiments were approved by the Medical Ethics Committee of Qingdao University Medical College, Qingdao, China (QUMC 2011-09).

Animal grouping. Sixty male healthy adult Wistar rats were randomly assigned into control $(n=6)$, sham operation $(n=6)$ and experimental $(n=48)$ groups. The experimental group was randomly subdivided into eight subgroups according to reperfusion time points $(3,6,12,24$ and $48 \mathrm{~h}$ and 3, 7 and 14 days), with 6 rats/subgroup. The animal models of middle cerebral artery occlusion/reperfusion (MCAO/R) were performed according to the Zea-Longa method (2).

Establishment of the animal model. The cerebral ischemia rat model was performed according to the Zea-Longa method (4). Following deep anesthesia of the rat bodies, the neck was opened in the midline, the right common carotid artery, external carotid artery (ECA), internal carotid artery (ICA) and the branches of ECA and ICA were separated, the ECA was sheared and a monofilament line was inserted to interrupt the flow through the middle cerebral artery (MCA). Reperfusion was permitted after $1 \mathrm{~h}$ of ischemia. The sham operation group only underwent separation of the blood vessels without any obstruction. The rats in the normal control group did not undergo any operation.

The rats developed a left-sided Horner syndrome. Flexing or circular crawling of the right anterior limb towards the right when the tail was lifted was considered a sign of a successful model. All the experimental procedures were performed in accordance with animal ethical standards.

Score of neurological function. When the tail was gently lifted $10 \mathrm{~cm}$ from the table, the normal rats extended their two forepaws on the table. According to the Bederson score standard (5), the scores were classified as 0-3.

Function test of balance and coordination. The rats were placed on an $80-\mathrm{cm}$ long and 2.4-cm wide balance beam, situated $10 \mathrm{~cm}$ above the ground. The experiment was performed according to the Altumbabic method (6). According to the Freeney score standard (7), the scores were classified as 0-5.

Measurement of forelimb placing. Referring to the Bland experiment (8), the investigator held the rat by the dorsal skin to suspend the limbs, with the vibrissae touching the table edge to detect ipsilateral forelimb activity. In response to the vibrissae lightly brushing the edge of the table, the forepaws of normal rats quickly touched the table, whereas the response of those with brain ischemic injury were affected to some extent. Each side of each rat was tested 10 times and the score was based on the percentage of times the forepaws touched the table edge. According to the Basso-Beattie-Bresnahan (BBB) score standard (9), the scores were classified as 0-21.

Preparation of paraffin sections. Six rats in each subgroup were deeply anesthetized by intraperitoneal injection of $10 \%$ chloral hydrate $(0.6 \mathrm{ml} / 100 \mathrm{~g})$ and perfused through the heart with phosphate-buffered solution, followed by $4 \%$ paraformaldehyde solution to fix the brain. The rats were then decapitated and middle brain tissue was collected. The tissue was continuously fixed in $4 \%$ paraformaldehyde for $2 \mathrm{~h}$, immersed in double-distilled water for $4 \mathrm{~h}$, underwent conventional gradient ethanol dehydration, were transparented in dimethylbenzene and embedded in paraffin. Coronal $6-\mu \mathrm{m}$ sections through the cortical area and the hippocampus were successively cut with a cryostat from the posterior of the optic chiasma and were then investigated with immunohistochemistry. The positive control section was provided by an immunohistochemistry agent and the negative control was set up by $0.01 \mathrm{~mol} / \mathrm{l} \mathrm{PBS}$ replacing the primary antibody to eliminate false-positive or false-negative results.

Cell apoptosis assay. The TUNEL staining method was employed to detect cell apoptosis. The procedure was performed according to the manufacturer's instructions (Roche Diagnostics GmbH). Following TUNEL staining, the sections were examined under a light microscope to observe apoptotic neurons in the cortex and hippocampus.

Immunohistochemistry assay. Rabbit anti-rat caspase-3, Bax and Bcl-2 immunohistochemistry kits were employed to detect the expression of the three proteins. Assay procedures were performed according to the manufacturer's instructions. Following immunohistochemical staining, the brain tissue sections were placed under the microscope to observe caspase-3-, Bax- and Bcl-2-positive neurons in the cortical area and the CA1, stratum lucidum (Slu) and molecular layer of the dentate gyrus (MoDG) of the hippocampus. The HPIAS-1000 Image Analysis system was used to calculate the total number of positive cells in the ischemic cortical area and the hippocampus.

Semiquantitive reverse transcriptase polymerase chain reaction (RT-PCR). The sequences of the caspase-3, Bax, Bcl-2 and $\beta$-actin genes were obtained from GeneBank. RT-PCR primers were designed by Primer Premier 5.0 software (Premier Biosoft International, CA, USA) to amplify gene mRNA. Total RNA was isolated by the TRIzol kit. RT-PCR was performed according to the manufacturer's instructions (Perkin Elmer). The RT-PCR product was analyzed by $1 \%$ agarose gel electrophoresis, images were captured by Bio-Gel analysis system, the absorbance of each band was measured with the analysis system, compared with the internal control $\beta$-actin and statistical analysis was performed between the two groups.

Statistical analysis. Data analyses were peformed with SPSS software, version 14.0 (SPSS Inc., Chicago, IL, USA). Data were compared with the $\chi^{2}$ test and are expressed as means \pm standard deviation. Analysis of variance (ANOVA) was used to compare variables within a group and between groups. $\mathrm{P}<0.05$ was considered to indicate a statistically significant difference. 
Table I. Ethology scores in the ischemia/reperfusion experimental and sham operation groups at different time points (mean \pm standard deviation).

\begin{tabular}{|c|c|c|c|c|c|c|c|}
\hline \multirow[b]{2}{*}{ Time points } & \multirow[b]{2}{*}{ No. } & \multicolumn{2}{|c|}{ Neurological function score } & \multicolumn{2}{|c|}{ Balance beam walking score } & \multicolumn{2}{|c|}{ Forelimb placing test (\%) } \\
\hline & & Sham & Experimental & Sham & Experimental & Sham & Experimental \\
\hline $3 \mathrm{~h}(\mathrm{I} / \mathrm{R})$ & 6 & $1.0 \pm 0.5$ & $3.0 \pm 0.3^{\mathrm{a}}$ & $1.0 \pm 0.4$ & $3.0 \pm 0.5^{\mathrm{a}}$ & $8.0 \pm 0.2$ & $5.0 \pm 0.2^{\mathrm{a}}$ \\
\hline $6 \mathrm{~h}(\mathrm{I} / \mathrm{R})$ & 6 & $0.0 \pm 0.0$ & $2.0 \pm 0.4$ & $0.0 \pm 0.0$ & $2.0 \pm 0.4$ & $8.0 \pm 0.3$ & $6.0 \pm 0.4^{\mathrm{a}}$ \\
\hline $12 \mathrm{~h}(\mathrm{I} / \mathrm{R})$ & 6 & $0.0 \pm 0.0$ & $2.0 \pm 0.2$ & $0.0 \pm 0.0$ & $2.0 \pm 0.3$ & $9.0 \pm 0.4$ & $7.0 \pm 0.3^{\mathrm{a}}$ \\
\hline $24 \mathrm{~h}(\mathrm{I} / \mathrm{R})$ & 6 & $0.0 \pm 0.0$ & $1.0 \pm 0.3$ & $0.0 \pm 0.0$ & $1.0 \pm 0.4$ & $9.0 \pm 0.5$ & $7.0 \pm 0.5^{\mathrm{a}}$ \\
\hline $48 \mathrm{~h}(\mathrm{I} / \mathrm{R})$ & 6 & $0.0 \pm 0.0$ & $1.0 \pm 0.2$ & $0.0 \pm 0.0$ & $1.0 \pm 0.3$ & $9.0 \pm 0.3$ & $8.0 \pm 0.3^{\mathrm{b}}$ \\
\hline $3 \mathrm{~d}(\mathrm{I} / \mathrm{R})$ & 6 & $0.0 \pm 0.0$ & $0.0 \pm 0.0$ & $0.0 \pm 0.0$ & $0.0 \pm 0.0$ & $9.0 \pm 0.4$ & $9.0 \pm 0.4$ \\
\hline $7 \mathrm{~d}(\mathrm{I} / \mathrm{R})$ & 6 & $0.0 \pm 0.0$ & $0.0 \pm 0.0$ & $0.0 \pm 0.0$ & $0.0 \pm 0.0$ & $9.0 \pm 0.2$ & $9.0 \pm 0.5$ \\
\hline $14 \mathrm{~d}(\mathrm{I} / \mathrm{R})$ & 6 & $0.0 \pm 0.0$ & $0.0 \pm 0.0$ & $0.0 \pm 0.0$ & $0.0 \pm 0.0$ & $9.0 \pm 0.3$ & $9.0 \pm 0.4$ \\
\hline
\end{tabular}

Neurological function score, balance beam walking score and forelimb placing test vs. sham operation group, ${ }^{\text {a }}<0.01$ and ${ }^{b} \mathrm{P}<0.05$ vs. sham operation group. I/R, ischemia/reperfusion; h, hours; d, days.

Table II. Basso, Beattie and Bresnahan (BBB) score following reperfusion at different time points in each group (mean \pm standard deviation).

\begin{tabular}{ccccc}
\hline & & \multicolumn{2}{c}{ Groups } \\
\cline { 3 - 5 } Time points & No. & Control & Sham & Experimental \\
\hline $3 \mathrm{~h}(\mathrm{I} / \mathrm{R})$ & 6 & $20.0 \pm 3.0$ & $18.0 \pm 1.0$ & $9.0 \pm 2.0^{\mathrm{a}}$ \\
$6 \mathrm{~h}(\mathrm{I} / \mathrm{R})$ & 6 & $20.0 \pm 3.0$ & $18.0 \pm 1.0$ & $11.0 \pm 2.0^{\mathrm{a}}$ \\
$12 \mathrm{~h}(\mathrm{I} / \mathrm{R})$ & 6 & $21.0 \pm 4.0$ & $19.0 \pm 1.0$ & $13.0 \pm 1.0^{\mathrm{a}}$ \\
$24 \mathrm{~h}(\mathrm{I} / \mathrm{R})$ & 6 & $20.0 \pm 3.0$ & $19.0 \pm 2.0$ & $17.0 \pm 1.0^{\mathrm{b}}$ \\
$48 \mathrm{~h}(\mathrm{I} / \mathrm{R})$ & 6 & $21.0 \pm 4.0$ & $20.0 \pm 2.0$ & $18.0 \pm 2.0^{\mathrm{b}}$ \\
$3 \mathrm{~d}(\mathrm{I} / \mathrm{R})$ & 6 & $20.0 \pm 3.0$ & $20.0 \pm 3.0$ & $19.0 \pm 2.0^{\mathrm{c}}$ \\
$7 \mathrm{~d}(\mathrm{I} / \mathrm{R})$ & 6 & $20.0 \pm 4.0$ & $20.0 \pm 3.0$ & $19.0 \pm 3.0^{\mathrm{c}}$ \\
$14 \mathrm{~d}(\mathrm{I} / \mathrm{R})$ & 6 & $21.0 \pm 3.0$ & $21.0 \pm 4.0$ & $20.0 \pm 4.0^{\mathrm{c}}$ \\
\hline
\end{tabular}

${ }^{a} \mathrm{P}<0.01$ vs. sham operation and control groups; ${ }^{\mathrm{b}} \mathrm{P}<0.05$, vs. the same group; ${ }^{\mathrm{c}} \mathrm{P}<0.05$ vs. sham operation and control groups. $\mathrm{I} / \mathrm{R}$, ischemia/reperfusion; h, hours; d, days.

\section{Results}

Neurological function score. Compared to the sham operation group, the resistance to side push on the paralyzed side was decreased following $3 \mathrm{~h}$ of reperfusion in the experimental subgroup, accompanied by anterior limb flexing, with a distinct score difference $(\mathrm{P}<0.01)$. The spontaneous rotation accompanied with walking for the 6-48 h subgroups after reperfusion, showed a distinct difference in the function scores. The neurological function gradually improved with reperfusion lasting 3-14 days (Table I).

Balance beam walking test. Compared to the sham operation group, when the experimental rats walked on a balance beam at $3 \mathrm{~h}$ following reperfusion, the anterior limb on the paralyzed side was not able to move forward, with a distinct difference in the function scores $(\mathrm{P}<0.01)$. At $6-48 \mathrm{~h}$ following reperfusion the rats were not able to walk on the balance beam, although they were able to sit on it, with a distinct difference in the scores. The motor ability significantly improved with reperfusion lasting 3-14 days (Table I).

Measurement of forelimb placing. Compared to the sham operation group, the positive percentage of the rats that were able to touch the table angle or edge with their anterior limbs at $3 \mathrm{~h}$ following reperfusion was extremely low $(\mathrm{P}<0.01)$, whereas that at 6-48 h reperfusion was notably increased, with a significant difference in the scores $(\mathrm{P}<0.05)$. The percentage following 3 days of reperfusion was identical to that of the sham operation and control groups (Table I).

$B B B$ score. Compared to the sham operation and control groups, the BBB score was distinctly decreased in the experimental group following reperfusion for 3-12 h $(\mathrm{P}<0.01)$ and 
Table III. Expression of TUNEL-positive neurons in the cortex and CA3 region of the hippocampus at different time points following ischemia/reperfusion (mean \pm standard deviation).

\begin{tabular}{|c|c|c|c|c|c|c|c|}
\hline \multirow{2}{*}{$\begin{array}{l}\text { Time } \\
\text { points }\end{array}$} & \multirow[b]{2}{*}{ No. } & \multicolumn{3}{|c|}{ Cortex } & \multicolumn{3}{|c|}{ Hippocampus } \\
\hline & & Control & Sham & Experimental & Control & Sham & Experimental \\
\hline $3 \mathrm{~h}(\mathrm{I} / \mathrm{R})$ & 6 & $5.72 \pm 1.10$ & $6.30 \pm 1.14$ & $8.75 \pm 1.12^{\mathrm{a}}$ & $5.50 \pm 1.21$ & $6.21 \pm 1.14$ & $8.64 \pm 1.17^{\mathrm{a}}$ \\
\hline $6 \mathrm{~h}(\mathrm{I} / \mathrm{R})$ & 6 & $6.36 \pm 1.17$ & $6.75 \pm 1.23$ & $13.63 \pm 2.27^{\mathrm{b}}$ & $6.21 \pm 1.34$ & $6.60 \pm 1.31$ & $13.34 \pm 2.83^{b}$ \\
\hline $12 \mathrm{~h}(\mathrm{I} / \mathrm{R})$ & 6 & $6.39 \pm 1.46$ & $7.18 \pm 1.35$ & $17.42 \pm 3.87^{\mathrm{b}}$ & $6.32 \pm 1.23$ & $7.04 \pm 1.41$ & $15.17 \pm 3.21^{\mathrm{b}}$ \\
\hline $24 \mathrm{~h}(\mathrm{I} / \mathrm{R})$ & 6 & $7.63 \pm 1.35$ & $7.57 \pm 1.65$ & $19.71 \pm 4.24^{\mathrm{b}}$ & $7.44 \pm 1.25$ & $7.11 \pm 1.19$ & $17.60 \pm 4.41^{\mathrm{b}}$ \\
\hline $48 \mathrm{~h}(\mathrm{I} / \mathrm{R})$ & 6 & $7.27 \pm 1.22$ & $8.08 \pm 1.74$ & $23.80 \pm 5.20^{\mathrm{b}}$ & $7.15 \pm 1.27$ & $8.00 \pm 1.36$ & $21.52 \pm 5.71^{\mathrm{b}, \mathrm{c}}$ \\
\hline $3 \mathrm{~d}(\mathrm{I} / \mathrm{R})$ & 6 & $6.84 \pm 1.46$ & $7.42 \pm 1.35$ & $16.64 \pm 3.56^{\mathrm{b}}$ & $6.70 \pm 1.39$ & $7.34 \pm 1.53$ & $15.61 \pm 3.52^{\mathrm{b}}$ \\
\hline $7 \mathrm{~d}(\mathrm{I} / \mathrm{R})$ & 6 & $6.27 \pm 1.32$ & $7.17 \pm 1.43$ & $12.32 \pm 3.34^{\mathrm{b}}$ & $5.20 \pm 1.25$ & $7.05 \pm 1.48$ & $11.31 \pm 2.01^{\mathrm{b}}$ \\
\hline $14 \mathrm{~d}(\mathrm{I} / \mathrm{R})$ & 6 & $5.53 \pm 1.28$ & $6.28 \pm 1.52$ & $7.12 \pm 1.31$ & $5.41 \pm 1.14$ & $6.21 \pm 1.31$ & $7.09 \pm 1.25$ \\
\hline
\end{tabular}

${ }^{\mathrm{a}} \mathrm{P}<0.05,{ }^{\mathrm{b}} \mathrm{P}<0.01$ vs. sham operation and control groups; ${ }^{\mathrm{c}} \mathrm{P}<0.01$, vs. the same group. I/R, ischemia/reperfusion; $\mathrm{h}$, hours; $\mathrm{d}$, days.
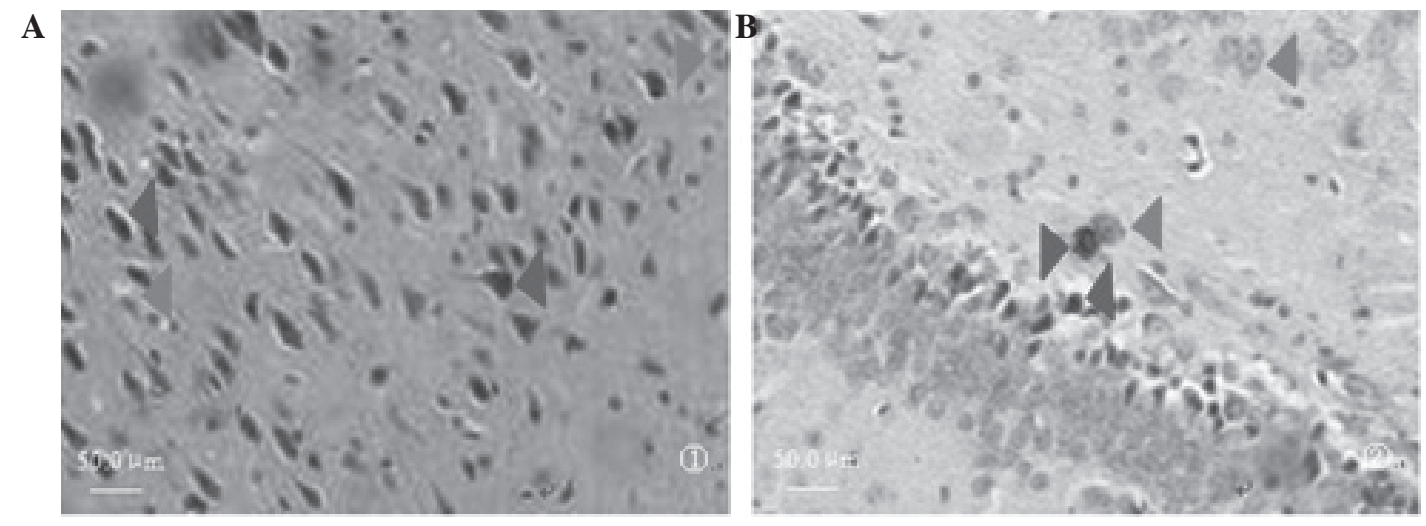

Figure 1. TUNEL staining of apoptotic neurons in (A) the cerebral cortex and (B) the hippocampal CA3 region. Apoptotic neurons are round, with intensely stained buff-coloured nuclei and shrunk, heavily stained cell membranes. Negative cells show deeply stained nuclei, lightly stained cytoplasm and maintained cell membrane intergrity. Magnification, x200.

24-48 $\mathrm{h}(\mathrm{P}<0.05)$. The differences in the scores were not significantly different following reperfusion for 3-14 days $(\mathrm{P}<0.05)$ (Table II).

Expression of TUNEL-positive neurons in ischemic regions. Compared to the sham operation group, the expression of TUNEL-positive neurons in the cortex and the CA3 region of the hippocampus was not significantly different $(\mathrm{P}<0.05)$ following reperfusion for $3 \mathrm{~h}$. The expression was markedly increased during reperfusion for 6 h-7 days, reaching a peak at $48 \mathrm{~h}(\mathrm{P}<0.01)$. The number of TUNEL-positive neurons following reperfusion for 14 days did not differ significantly between the groups $(\mathrm{P}<0.05)$ (Table III, Fig. 1A and B).

RT-PCR of apoptosis-related gene mRNA in the cerebral cortex and hippocampus. RT-PCR demonstrated positive caspase-3, Bax, Bcl-2 and $\beta$-actin mRNA expression in the cerebral cortex and hippocampus of the rats in each group (Fig. 2).

Expression of caspase-3-, Bax- and Bcl-2-positive neurons in the cerebral cortex and hippocampus. Compared to the sham operation group, the expression of caspase-3-positive

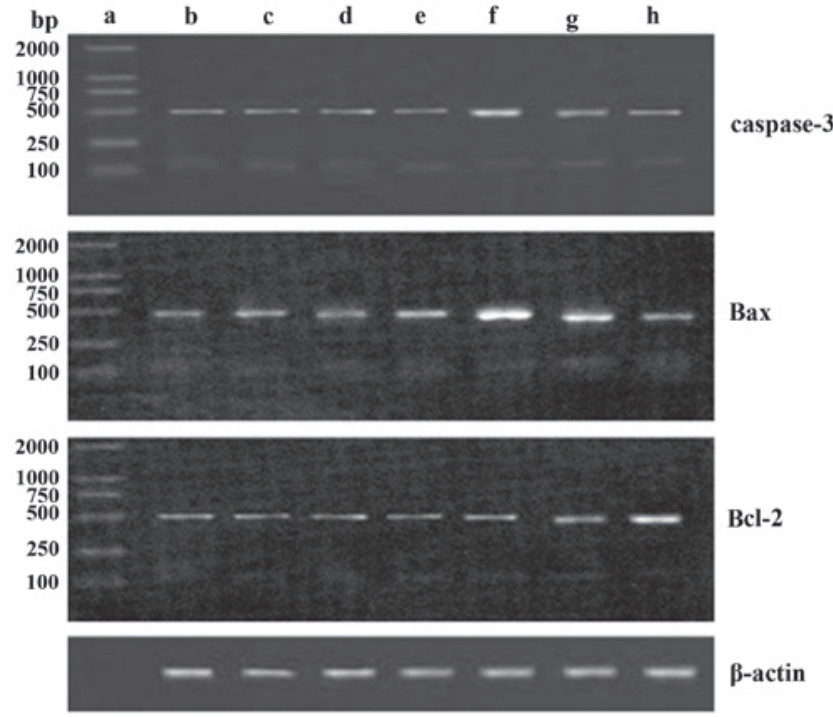

Figure 2. Reverse transcriptase polymerase chain reaction (RT-PCR) results for caspase-3, Bax, Bcl-2 and $\beta$-actin mRNA in each group at various time points. (a) Marker; (b) Sham operation group; (c) $3 \mathrm{~h}$; (d) $12 \mathrm{~h}$; (e) $24 \mathrm{~h}$; (f) $48 \mathrm{~h}$; (g) 7 days and (h) 14 days following ischemia/reperfusion in the experimental group. 
a
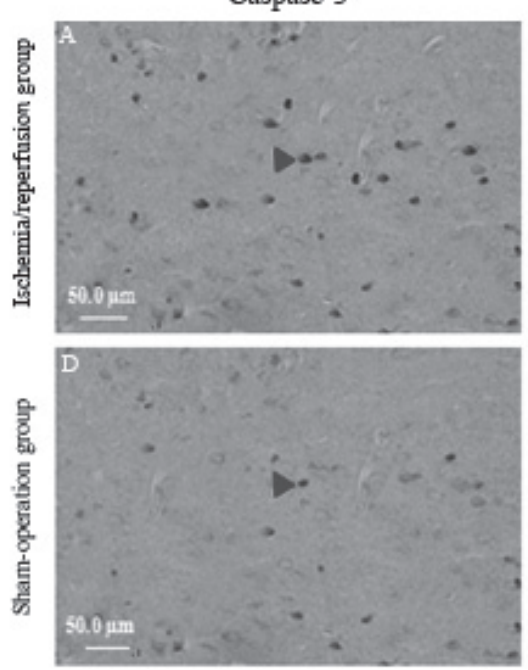

b
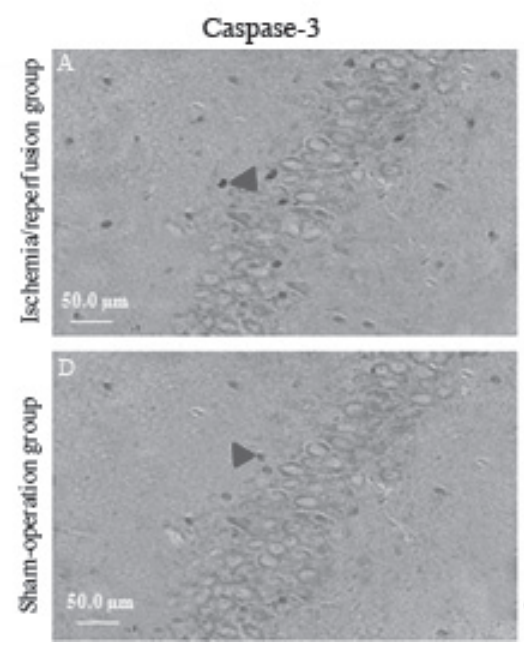

Bax
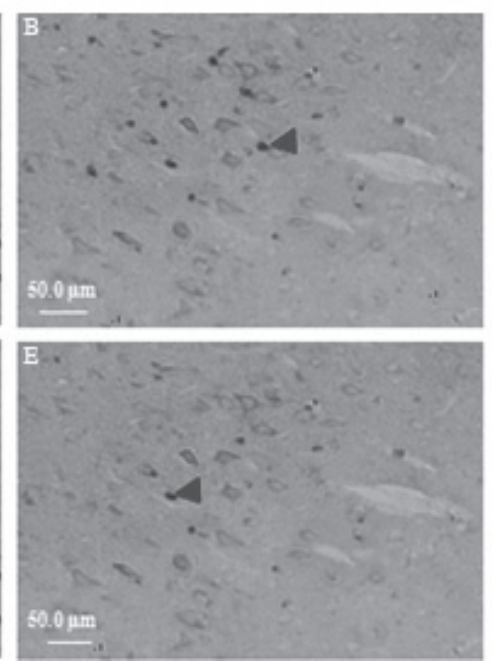

Bax
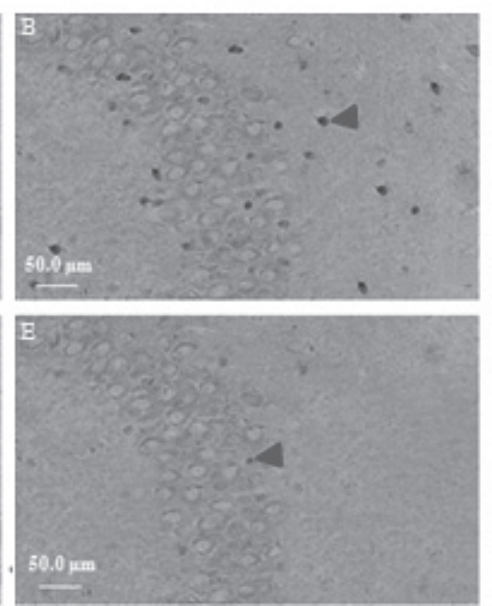

Bcl-2
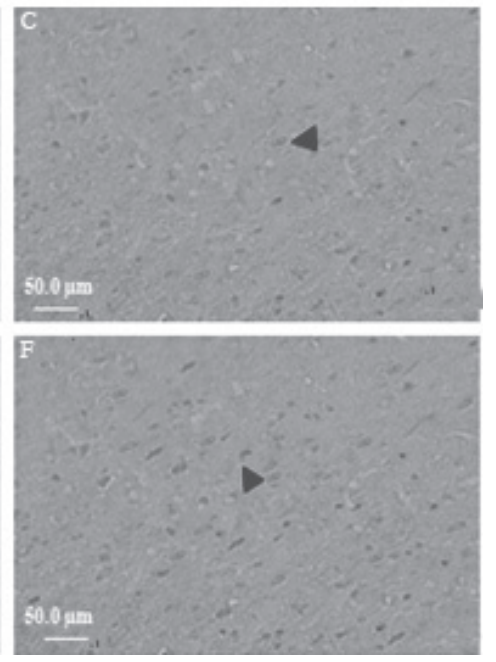

Bcl-2
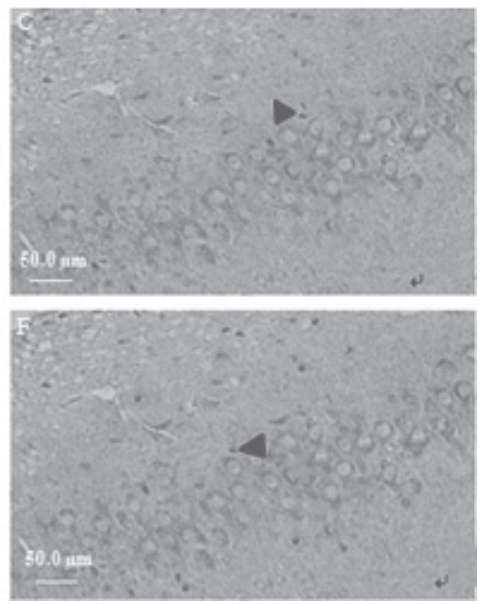

Figure 3. (a) Expression of caspase-3, Bax and Bcl-2 in the cerebral cortex at $12 \mathrm{~h}$ following reperfusion. (A-C) Sham operation group; (D-F) ischemia/reperfusion group. (A) The number of caspase-3-positive neurons was distinctly increased compared to (D); (B) the number of Bax-positive neurons was higher compared to (E); (C) The number of Bcl-2-positive neurons was lower compared to (F). Immunohistochemichal staining, A,B,D and E: magnification, x200; E and F: magnification, x100. (b) Expression of caspase-3, Bax and Bcl-2 in the hippocampus at $12 \mathrm{~h}$ following reperfusion. (A-C) Sham operation group; (D-F) Ischemia/reperfusion group. (A) The number of caspase-3-positive neurons was distinctly increased compared to (D); (B) the number of Bax-positive neurons was higher compared to (E); (C) the number of Bcl-2-positive neurons was lower compared to (F). Immunohistochemichal staining, magnification, x200.

neurons was distinctly different at $3 \mathrm{~h}$ following reperfusion in the cerebral cortex and the CA1 region of the hippocampus $(\mathrm{P}<0.05)$ and was further increased following reperfusion for $6 \mathrm{~h}-7$ days (Fig. 3aA and bA), reaching a peak at $48 \mathrm{~h}(\mathrm{P}<0.01)$, decreasing approximately to sham operation or normal control group levels at 14 days (Table IV).

Compared to the sham operation group, the expression of Bax-positive neurons did not differ significantly at $3 \mathrm{~h}$ or 14 days of reperfusion in the cerebral cortex and the Slu of the hippocampus $(\mathrm{P}<0.05)$, although it was higher at $6,12,24$, $48 \mathrm{~h}$ and 7 days, with a peak at $48 \mathrm{~h}$ after ischemia/reperfusion $(\mathrm{P}<0.01)$ (Fig. $3 \mathrm{aB}$ and bB, Table V).

Compared to the sham operation group, the expression of Bcl-2-positive neurons did not differ at $3 \mathrm{~h}$ or 14 days of reperfusion in the cerebral cortex and the Slu of the hippocampus $(\mathrm{P}<0.05)$, although it was lower at $6,12,24,48 \mathrm{~h}$ and 7 days, with the lowest at $48 \mathrm{~h}$ after ischemia/reperfusion $(\mathrm{P}<0.01)$ (Fig. $3 \mathrm{aC}$ and bC, Table VI).

\section{Discussion}

It is crucial to establish an $\mathrm{MCAO} / \mathrm{R}$ animal model according to the clinical characteristics of a human cerebral natural infarct. The model characteristics are as follows: i) single damage mechanism that is easy to study; ii) simple method, small wound, easy to control condition, stable infarct site, and distinct symptomatic reaction and high achievement ratio; iii) uniformity of cerebral infarction and good reproducibility; and iv) necrotic brain tissue following injury, with a similar pathophysiological process to clinical cerebral ischemia. In this study, a rat cerebral ischemia/reperfusion injury model was established according to the Zea-Longa method (4). Following cerebral ischemia for $1 \mathrm{~h}$, the rats developed severe nervous and behavioral functional impairment symptoms, indicating the establishment of a successful model.

The Bederson (5) score method was employed for qualitative and semiquantitative evaluation, with particular emphasis 
Table IV. Expression of caspase-3-positive neurons at different time points in the cortex and the hippocampus following ischemia/reperfusion (mean \pm standard deviation).

\begin{tabular}{|c|c|c|c|c|c|c|c|}
\hline \multirow{2}{*}{$\begin{array}{l}\text { Time } \\
\text { points }\end{array}$} & \multirow[b]{2}{*}{ No. } & \multicolumn{3}{|c|}{ Cortex } & \multicolumn{3}{|c|}{ Hippocampus } \\
\hline & & Control & Sham & Experimental & Control & Sham & Experimental \\
\hline 3 h (I/R) & 6 & $5.51 \pm 1.01$ & $6.26 \pm 1.10$ & $8.80 \pm 1.08^{\mathrm{a}}$ & $5.48 \pm 1.03$ & $6.15 \pm 1.06$ & $8.71 \pm 1.00^{\mathrm{a}}$ \\
\hline $6 \mathrm{~h}(\mathrm{I} / \mathrm{R})$ & 6 & $5.23 \pm 1.24$ & $6.13 \pm 1.12$ & $13.91 \pm 2.83^{b}$ & $5.20 \pm 1.26$ & $6.08 \pm 1.12$ & $11.11 \pm 2.01^{\mathrm{b}}$ \\
\hline 12 h (I/R) & 6 & $6.38 \pm 1.17$ & $7.16 \pm 1.41$ & $15.40 \pm 3.15^{\mathrm{b}}$ & $6.34 \pm 1.18$ & $7.10 \pm 1.14$ & $13.23 \pm 2.23^{b}$ \\
\hline $24 \mathrm{~h}(\mathrm{I} / \mathrm{R})$ & 6 & $7.54 \pm 1.10$ & $8.10 \pm 1.26$ & $18.25 \pm 3.45^{\mathrm{b}}$ & $7.16 \pm 1.20$ & $8.03 \pm 1.23$ & $17.60 \pm 3.07^{\mathrm{b}}$ \\
\hline 48 h (I/R) & 6 & $7.12 \pm 1.41$ & $8.12 \pm 1.71$ & $20.08 \pm 3.78^{\mathrm{b}, \mathrm{c}}$ & $7.33 \pm 1.35$ & $8.07 \pm 1.54$ & $19.10 \pm 3.31^{\mathrm{b}, \mathrm{c}}$ \\
\hline $3 \mathrm{~d}(\mathrm{I} / \mathrm{R})$ & 6 & $6.51 \pm 1.36$ & $7.34 \pm 1.50$ & $15.67 \pm 2.32^{\mathrm{b}}$ & $6.35 \pm 1.26$ & $7.21 \pm 1.27$ & $13.09 \pm 2.17^{\mathrm{b}}$ \\
\hline $7 \mathrm{~d}(\mathrm{I} / \mathrm{R})$ & 6 & $5.13 \pm 1.25$ & $6.23 \pm 1.66$ & $12.70 \pm 2.11^{\mathrm{b}}$ & $6.10 \pm 1.27$ & $6.19 \pm 1.20$ & $10.38 \pm 2.25^{\mathrm{b}}$ \\
\hline $14 \mathrm{~d}(\mathrm{I} / \mathrm{R})$ & 6 & $5.49 \pm 1.28$ & $6.10 \pm 1.09$ & $8.11 \pm 1.19$ & $5.05 \pm 1.22$ & $6.02 \pm 1.21$ & $8.03 \pm 1.12$ \\
\hline
\end{tabular}

${ }^{\mathrm{a}} \mathrm{P}<0.05,{ }^{\mathrm{b}} \mathrm{P}<0.01$ vs. sham operation and control groups; ${ }^{\mathrm{c}} \mathrm{P}<0.05$ vs. the same group. I/R, ischemia/reperfusion; h, hours; $d$, days.

Table V. Expression of Bax-positive neurons at different time points in the cortex and the hippocampus following ischemia/reperfusion (mean \pm standard deviation).

\begin{tabular}{|c|c|c|c|c|c|c|c|}
\hline \multirow{2}{*}{$\begin{array}{l}\text { Time } \\
\text { points }\end{array}$} & \multirow[b]{2}{*}{ No. } & \multicolumn{3}{|c|}{ Cortex } & \multicolumn{3}{|c|}{ Hippocampus } \\
\hline & & Control & Sham & Experimental & Control & Sham & Experimental \\
\hline $3 \mathrm{~h}(\mathrm{I} / \mathrm{R})$ & 6 & $5.23 \pm 1.22$ & $6.47 \pm 1.05$ & $8.70 \pm 1.03^{\mathrm{a}}$ & $5.20 \pm 1.28$ & $6.35 \pm 1.11$ & $8.59 \pm 1.01^{\mathrm{a}}$ \\
\hline $6 \mathrm{~h}(\mathrm{I} / \mathrm{R})$ & 6 & $6.27 \pm 1.21$ & $7.23 \pm 1.24$ & $11.53 \pm 2.11^{\mathrm{a}}$ & $6.21 \pm 1.34$ & $7.12 \pm 1.34$ & $10.23 \pm 2.07^{\mathrm{a}}$ \\
\hline 12 h (I/R) & 6 & $7.34 \pm 1.45$ & $8.51 \pm 1.51$ & $12.33 \pm 3.10^{\mathrm{a}}$ & $6.42 \pm 1.37$ & $8.49 \pm 1.08$ & $12.23 \pm 2.12^{\mathrm{a}}$ \\
\hline $24 \mathrm{~h}(\mathrm{I} / \mathrm{R})$ & 6 & $6.33 \pm 1.37$ & $7.71 \pm 1.49$ & $15.61 \pm 3.14^{b}$ & $5.31 \pm 1.27$ & $7.60 \pm 1.14$ & $14.38 \pm 3.21^{\mathrm{b}}$ \\
\hline $48 \mathrm{~h}(\mathrm{I} / \mathrm{R})$ & 6 & $7.46 \pm 1.32$ & $8.81 \pm 1.75$ & $21.16 \pm 3.24^{\mathrm{b}, \mathrm{c}}$ & $6.43 \pm 1.43$ & $7.27 \pm 1.31$ & $19.08 \pm 3.32^{b, c}$ \\
\hline $3 \mathrm{~d}(\mathrm{I} / \mathrm{R})$ & 6 & $6.12 \pm 1.54$ & $8.37 \pm 1.46$ & $12.94 \pm 3.31^{\mathrm{a}}$ & $6.10 \pm 1.35$ & $8.02 \pm 1.43$ & $11.12 \pm 3.71^{\mathrm{a}}$ \\
\hline $7 \mathrm{~d}(\mathrm{I} / \mathrm{R})$ & 6 & $5.37 \pm 1.48$ & $7.54 \pm 1.32$ & $10.62 \pm 2.15^{\mathrm{a}}$ & $5.28 \pm 1.21$ & $7.36 \pm 1.17$ & $9.82 \pm 2.20^{\mathrm{a}}$ \\
\hline $14 \mathrm{~d}(\mathrm{I} / \mathrm{R})$ & 6 & $5.20 \pm 1.35$ & $6.35 \pm 1.06$ & $7.18 \pm 1.00$ & $5.13 \pm 1.36$ & $6.21 \pm 1.06$ & $5.02 \pm 1.01$ \\
\hline
\end{tabular}

${ }^{\mathrm{a}} \mathrm{P}<0.05,{ }^{\mathrm{b}} \mathrm{P}<0.01$ vs. sham operation and control groups; ${ }^{\mathrm{C}} \mathrm{P}<0.05$ vs. the same group. I/R, ischemia/reperfusion; h, hours; $d$, days.

Table VI. Expression of Bcl-2-positive neurons at various time points in the cortex and the hippocampus following ischemia/reperfusion (mean \pm standard deviation).

\begin{tabular}{|c|c|c|c|c|c|c|c|}
\hline \multirow{2}{*}{$\begin{array}{l}\text { Time } \\
\text { points }\end{array}$} & \multirow[b]{2}{*}{ No. } & \multicolumn{3}{|c|}{ Cortex } & \multicolumn{3}{|c|}{ Hippocampus } \\
\hline & & Control & Sham & Experimental & Control & Sham & Experimental \\
\hline $3 \mathrm{~h}(\mathrm{I} / \mathrm{R})$ & 6 & $24.13 \pm 1.32$ & $23.32 \pm 1.21$ & $21.13 \pm 1.06^{\mathrm{a}}$ & $24.06 \pm 1.35$ & $23.17 \pm 1.28$ & $20.08 \pm 2.63^{\mathrm{a}}$ \\
\hline $6 \mathrm{~h}(\mathrm{I} / \mathrm{R})$ & 6 & $21.75 \pm 1.54$ & $21.47 \pm 1.27$ & $19.23 \pm 3.42^{\mathrm{a}}$ & $21.10 \pm 1.51$ & $22.23 \pm 1.51$ & $18.07 \pm 2.31^{\mathrm{a}}$ \\
\hline $12 \mathrm{~h}(\mathrm{I} / \mathrm{R})$ & 6 & $22.21 \pm 1.47$ & $20.55 \pm 1.40$ & $18.12 \pm 3.24^{\mathrm{a}}$ & $22.18 \pm 1.42$ & $21.07 \pm 1.21$ & $16.17 \pm 3.07^{\mathrm{a}}$ \\
\hline $24 \mathrm{~h}(\mathrm{I} / \mathrm{R})$ & 6 & $23.47 \pm 1.44$ & $21.29 \pm 1.42$ & $12.73 \pm 2.16^{\mathrm{b}}$ & $22.10 \pm 1.46$ & $20.11 \pm 1.32$ & $11.62 \pm 3.32^{b}$ \\
\hline $48 \mathrm{~h}(\mathrm{I} / \mathrm{R})$ & 6 & $21.29 \pm 1.35$ & $20.15 \pm 1.30$ & $9.94 \pm 2.17^{\mathrm{b}, \mathrm{c}}$ & $21.21 \pm 1.41$ & $20.14 \pm 1.26$ & $9.75 \pm 2.43^{b, c}$ \\
\hline $3 \mathrm{~d}(\mathrm{I} / \mathrm{R})$ & 6 & $22.84 \pm 1.23$ & $21.51 \pm 1.34$ & $17.41 \pm 2.13^{\mathrm{a}}$ & $23.70 \pm 1.50$ & $21.24 \pm 1.33$ & $17.12 \pm 4.01^{\mathrm{a}}$ \\
\hline $7 \mathrm{~d}(\mathrm{I} / \mathrm{R})$ & 6 & $23.68 \pm 1.58$ & $22.23 \pm 1.15$ & $19.21 \pm 3.01^{\mathrm{a}}$ & $22.43 \pm 1.57$ & $22.20 \pm 1.19$ & $19.01 \pm 2.38^{\mathrm{a}}$ \\
\hline $14 \mathrm{~d}(\mathrm{I} / \mathrm{R})$ & 6 & $21.36 \pm 1.47$ & $21.17 \pm 1.38$ & $20.98 \pm 1.04$ & $21.21 \pm 1.43$ & $21.11 \pm 1.20$ & $20.16 \pm 1.31$ \\
\hline
\end{tabular}

${ }^{a} \mathrm{P}<0.05,{ }^{b} \mathrm{P}<0.01$ vs. sham operation and control groups; ${ }^{\mathrm{C}} \mathrm{P}<0.05$ vs. the same group. I/R, ischemia/reperfusion; $\mathrm{h}$, hours; $d, d a y s$. 
on motor function evaluation. The balance beam walking assay evaluated motor reaction speed, fine motor coordination ability and balance under surrounding stimuli. The anterior limb-placing assay evaluated reaction ability, vision, touch and proprioceptive sensation. A normal reaction was considered the anterior limb immediately gripping the tabletop and the behavioral response of injured rats was retardation. The results demonstrated that the scores of neurological function and balance beam walking test were decreased from 3 to 1 at 6-48 h following cerebral ischemia/reperfusion in experimental rats, although that of the forelimb placing assay was distinctly increased at 6-12 h of reperfusion, which indicates that reperfusion for 6-48 $\mathrm{h}$ is the time window causing injury of the cortical and hippocampal vulnerable regions. Motor behavior functional impairment was closely correlated with reperfusion injury.

The order of vulnerability of the hippocampal subsectors to global ischemia is as follows: $\mathrm{CAl}$, hilus $>\mathrm{CA} 2>\mathrm{CA} 3>$ dentate gyrus (10). A recent study by Xie et al (11) demonstrated that the hippocampal CA1 area ipsilateral to the occluded MCA may undergo delayed neuronal death and the process may be due to the spread of injury signals through the astrocytic gap junction from the infarct region to the remote hippocampus. Neurological impairment mediated by neuron apoptosis was confirmed by the experiment. The results demonstrated that TUNEL-positive cells reached a peak expression at $48 \mathrm{~h}$ following reperfusion in the cortical area and the CA3 of the hippocampus, which is in accordance with the results of Ou Yang et al (12) and indicates that the energy did not exhaust but inhibited protein metabolism, leading to neuron apoptosis $\sim 48 \mathrm{~h}$ after reperfusion. The time point of $48 \mathrm{~h}$ after reperfusion may be the key time circumscription resulting in pathological changes of the cortex and hippocampus.

Caspase- 3 is an important protein in the apoptotic enzyme cascade and regulates neuron apoptosis. Our results have demonstrated that the expression of caspase- 3 and neuron apoptosis reached a peak at $\sim 48 \mathrm{~h}$ after reperfusion in the cortex and the CA1 and CA3 regions of the hippocampus. The disposition of the two was considered a coincidence and was consistent with the experiment of Choi et al (13). This finding indicated that ischemia/reperfusion injury enhanced caspase-3 activity or induced its expression, promoting neuron apoptosis.

The Bax protein exerts an important effect in the regulation of cell apoptosis. High Bax expression levels and formation of homo- or heterodimers with Bcl-2 may lead to cell death $(14,15)$. It was previously demonstrated that Bax is highly expressed at 24-72 $\mathrm{h}$ following reperfusion (16). Our results have demonstrated that the expression of Bax was distinctly increased at $48 \mathrm{~h}$ following reperfusion in the cortical area and the Slu of the hippocampus, which was consistent with those results. The time and disposition of Bax expression coincided with neuron TUNEL positivity. This indicated that the expression of Bax altered the balance between the genes promoting and those inhibiting apoptosis, inducing or accelerating neuron apoptosis and possibly increasing the activity of caspase-3.
Bcl-2 is the key cell apoptosis inhibitory protein. Its regulatory effect depends on its expression, although the Bcl-2/Bax ratio is also considered a key factor. When the $\mathrm{Bcl}-2 / \mathrm{Bax}$ ratio increases, cell apoptosis is inhibited and when the ratio is decreased, apoptosis is promoted. The expression levels of $\mathrm{Bcl}-2$ were low at $48 \mathrm{~h}$ following reperfusion in the cortex and the MoDG of the hippocampus, in accordance with the results of Qin et al (15). This may be the reason that the low levels of Bcl-2 expression and the decrease of the Bcl-2/Bax ratio resulted in neuron apoptosis.

In conclusion, the successful establishment of a cerebral ischemia/reperfusion injury animal model and the different expressions of caspase-3, Bax and BcL-2 following ischemia/reperfusion may provide important reference evidence on the etiopathogenesis of clinical cerebral thrombosis.

\section{References}

1. Candelario-Jalil E: Injury and repair mechanisms in ischemic stroke: considerations for the development of novel neurotherapeutics. Curr Opin Investig Drugs 10: 644-654, 2009.

2. Li Q, Zhang R, Guo YL and Mei YW: Effect of neuregulin on apoptosis and expressions of STAT3 and GFAP in rats following cerebral ischemic reperfusion. J Mol Neurosci 37: 67-73, 2009.

3. Li Q, Zhang R, Ge YL, Mei YW and Guo YL: Effects of neuregulin on expression of MMP-9 and NSE in brain of ischemia/reperfusion rat. J Mol Neurosci 38: 207-215, 2009.

4. Longa EZ, Weistein PR, Calson S, et al: Reversible middle cerebral artery occlusion without craniectomy in rats. Stroke 20: 84-89, 1989.

5. Bederson JB, Pitts LH, Tsuji M, et al: Rat middle cerebral artery occlusion: evaluation of the model and development of a neurologic examination. Stroke 17: 472-476, 1986.

6. Altumbabic M, Peeling J, Del Bigio MR, et al: Intracerebral hemorrhage in the rat: effects of hematoma aspiration. Stroke 29: 1917-1922, 1998 .

7. Freeney DM, Boyeson MG, Linn RT, et al: Responses to cortical injury: I. Methodology and local effects of contusions in the rat. Brain Res 211: 67-77, 1981.

8. Bland ST, Schallert T, Strong R, et al: Early exclusive use of the affected forelimb after moderate transient focal ischemia in rat: function and anatomic outcome. Stroke 31: 1144-1152, 2000.

9. Basso DM, Beattie MS and Bresnahan JC: A sensitive and reliable locomotor rating scale for open filed testing in rats. J Neurotrauma 12: 1-21, 1995.

10. Schmidt-Kastner R and Freund TF: Selective vulnerability of the hippocampus in brain ischemia. Neuroscience 40: 599-636, 1991.

11. Xie M, Yi C, Luo X, et al: Glial gap junctional communication involvement in hippocampal damage after middle cerebral artery occlusion. Ann Neurol 70: 121-132, 2011.

12. Ou-Yang FL, Zhou XZ, Fang SZ, Cai YQ and Li H: Long-term behavioral and ultrastructural alterations following hypoxic-ischemic brain damage in neonatal rats. Zhongguo Dang Dai Er Ke Za Zhi 14: 380-384, 2012 (In Chinese).

13. Choi JY, Kim BM, Kim YJ, et al: Hypoxia/reoxygenation-induced cytotoxicity in cultured human lymphocytes. Biochem Biophys Res Commun 352: 366-371, 2007.

14. Haddad JJ: The role of Bax/Bcl-2 and pro-caspase peptides in hypoxia/reperfusion-dependent regulation of MAPK (ERK): discordant proteomic effect of MAPK (p38). Protein Pept Lett 14: 361-371, 2007.

15. Qin AP, Zhang HL and Qin ZH: Mechanisms of lysosomal proteases participating in cerebral ischemia-induced neuronal death. Neurosci Bull 24: 117-123, 2008.

16. Xing B, Chen H, Zhang M, Zhao D, Jiang R, Liu X and Zhang S: Ischemic postconditioning inhibits apoptosis after focal cerebral ischemia/reperfusion injury in the rat. Stroke 39: 2362-2369, 2008. 